\title{
ANÁLISE SUBJETIVA DE FATORES PRÉ-ONCOGÊNICOS EM PACIENTES COM NEOPLASIAS DE CABEÇA E PESCOÇO, DE UM HOSPITAL DO SUL CATARINENSE
}

\section{SUBJECTIVE ANALYSIS OF PRE-ONCOGENIC FACTORS IN HEAD AND NECK CANCER IN A HOSPITAL IN SANTA CATARINA}

\author{
Juliana Milioli VOLTOLINI ${ }^{1}$ \\ julimilioli@hotmail.com \\ Ângela Catarina MARAGNO² \\ acmaragno@gmail.com.
}

Priscyla Waleska Targino de Azevedo SIMÕES 3

pritsimoes@gmail.com

\section{RESUMO}

Introdução: Mais de 550 mil casos de tumor de cabeça e pescoço surgem anualmente, e estes constituem a $5^{a}$ neoplasia mais prevalente no mundo. As neoplasias de cabeça e pescoço têm etiologia multifatorial, onde se destacam como principais agentes pré-oncológicos, segundo a literatura, o tabaco, álcool, HPV, exposição ao sol e o próprio envelhecimento. As lesões neoplásicas geralmente são detectadas tardiamente por serem assintomáticas, e em decorrência disso os tumores são descobertos em uma fase muito avançada, deixando graves sequelas e um baixo índice de sobrevida. Métodos: Aplicaram-se questionários em 35 pacientes com diagnóstico de tumor de cabeça e pescoço, e em 35 pacientes sem neoplasias diagnosticadas. O questionário avaliou os fatores pré-oncogênicos de cada grupo. Resultados: Observou-se dentre os pacientes com neoplasia: $71,4 \%$ sendo do sexo masculino; $94,3 \%$ caucasianos; $77,1 \%$ tendo histórico de câncer na família; 73,5\% já fizeram uso de tabaco; 37,1\% foram etilistas e 86,7\% tiveram um alto índice de radiação solar. Já no grupo de pacientes sem neoplasias, foi observado: $45,7 \%$ sendo do sexo masculino; $82,9 \%$ caucasianos; $65,7 \%$ tendo histórico de câncer na família; 39,3\% fizeram uso de tabaco; 8,6\% foram etilistas e 44,1\% tiveram um alto índice de radiação solar. Conclusão: Mediante o que foi pesquisado, pode-se afirmar que a maioria dos pacientes com neoplasias possuíam fatores pré-oncogênicos elevados. Já no grupo sem neoplasias diagnosticadas, encontrou-se um índice menor durante a pesquisa.

DESCRITORES: NEOPLASIAS DE CABEÇA E PESCOÇO; FATORES PRÉ-ONCOLÓGICOS; ONCOLOGIA.

1 Universidade do Extremo Sul Catarinense (UNESC), SC, Brasil - Pós Graduada em Odontologia Hospitalar - Hospital Israelita Albert Einstein (HIAE), SP

2 Universidade do Extremo Sul Catarinense (UNESC), SC, Brasil - Mestre em Radiologia - Docente do curso de odontologia da Unesc

3 Universidade Federal do ABC (UFABC), SP, Brasil - Doutora em Ciências da Saúde - Docente do curso de Engenharia Biomédica da UFABC. Centro de Engenharia, Modelagem e Ciências Sociais Aplicadas, Universidade Federal do ABC (UFABC).. 
ISSN 1983-5183

\section{ABSTRACT}

Introduction: More than 550.000 cases of head and neck tumors are discovered annually, being the 5th most prevalent neoplasm in the world. The number of tumor cases has been steadily increasing over the last few years around the world. The head and neck neoplasms have a multifactorial etiology, in which tobacco, alcohol, HPV, exposure to the sun and aging standout as the main pre-oncological agents. Neoplastic lesions are usually discovered too late, since they are asymptomatic, and as a result tumors are discovered at a very advanced stage, leaving severe sequelae and a low survival rate. Methods: Questionnaires were applied to 35 patients with diagnosis of head and neck tumors and to 35 patients without diagnosed neoplasms. This questionnaire evaluated the pre-oncogenic factors of each group. Results: It was observed among the patients with neoplasia: $71.4 \%$ were male; $94.3 \%$ Caucasians; $77.1 \%$ reported having a family history of cancer; $73.5 \%$ of patients have used tobacco; $37.1 \%$ reported having already been alcoholic drinker sand $86.7 \%$ had a high index of solar radiation. In the group of patients without neoplasias, $45.7 \%$ were male; $82.9 \%$ Caucasians; $65.7 \%$ reported having a family history of cancer; $39.3 \%$ of the patients have already used tobacco; $8.6 \%$ reported having already been alcoholic drinker sand $44.1 \%$ had a high index of solar radiation. Conclusion: Based on this search, it can be stated that the majority of patients with neoplasias had high pre-oncogenic factors. In the group without diagnosed neoplasms, a lower index was found during the study.

DESCRIPTORS: HEAD AND NECK NEOPLASMS; PRE-ONCOLOGICAL FACTORS; MEDICAL ONCOLOGY

\section{INTRODUÇÃO}

O câncer de cabeça e pescoço é a $5^{\circ}$ neoplasia mais frequente no mundo'. Envolvem-se nesta classificação de tumor de cabeça e pescoço os tumores presentes na região da cavidade oral, faringe, laringe, cavidade nasal, seios paranasais, tireoide e glândulas salivares ${ }^{2,3}$. Dá-se a nomenclatura carcinógeno para as substâncias que agem de modo direto ou indireto nas distintas fases da carcinogênese, que pode vir a ser provocada por uma variedade de agentes diferentes entre si, classificados como: agentes químicos, físicos, biológicos e genéticos ${ }^{4}$.

Pode-se citar, dentre os agentes químicos mais conhecidos atualmente, o tabaco e o álcool. O tabaco pode levar o DNA celular a obter um dano perpétuo, sendo que este processo ocorre durante a síntese do mesmo ${ }^{4}$. O uso do tabaco está intimamente relacionado com $80 \%$ do aparecimento de câncer bucal, pois contém mais de 60 substâncias ditas como carcinogênicas, dentre elas, nitrosaminas e também hidrocarbonetos policíclicos carcinogênicos genotóxicos, que conseguem elevar o risco da ocorrência de tal patologia, induzindo mutações pela modificação do perfil molecular 5 . O cigarro, vinculado ao uso do álcool, pode vir a aumentar 40 vezes a chance do aparecimento do carcinoma epidermoide6.

O álcool pode gerar mudanças na endocitose, produzindo uma redução da área citoplasmática e fazendo com que haja uma elevação na permeabilidade da membrana celular ${ }^{5}$.

Há um aumento do aparecimento de câncer em pessoas de idade mais avançada. Os tumores foram fragmentados em duas classes, que dizem respeito às idades em que ocorrem as maiores incidências. Os tumores surgem com maior incidência por volta dos 50 anos de idade, e correspondem à primeira classe. O segundo grupo é dividido em duas partes, com dois picos de incidência, antes dos 35 anos e após os 50 anos. É mais comum a ocorrência de tumores na primeira classe?. 
ISSN 1983-5183

Outro fator que está associado ao aparecimento de lesões escamosas bucais é o HPV, apresentando malignidade ou não. O seu local de aparecimento preferencial vem a ser na orofaringe $^{8}$. Mais de 100 tipos de HPV já foram encontrados em diversas lesões e podem ser divididos em alto risco (oncogênicos) e baixo risco (não oncogênicos) ${ }^{9}$.

A radiação ultravioleta também tem um papel importante na carcinogênese, podendo gerar alterações celulares, fazendo com que futuramente apareça um tumor ${ }^{10}$. Geralmente, a exposição contínua ao sol está ligada ao aparecimento de queilite actínica, leucoplasia e carcinoma epidermoide de lábio"1.

Os fatores pré-oncológicos estão presentes no cotidiano, apresentando-se de variadas formas. Sendo assim, são necessários cada vez mais estudos para que se possa estabelecer um perfil de pacientes mais susceptíveis e compreender melhor a influência dos fatores químicos, físicos e biológicos que envolvem a formação de neoplasias de cabeça e pescoço.

A importância deste estudo constituiu-se em identificar a presença de fatores préoncológicos nos pacientes com diagnóstico de neoplasias de cabeça e pescoço, de um Hospital do Sul Catarinense.

\section{MÉTODOS}

Realizou-se um estudo transversal descritivo que avaliou pacientes em tratamento de neoplasias de cabeça e pescoço, no Hospital São José, em Criciúma - SC, representando o grupo-amostra. O grupocontrole foi formado por pacientes em atendimento nas clínicas integradas da Universidade do Extremo Sul Catarinense (UNESC), que não apresentavam neoplasias de cabeça e pescoço diagnosticadas. Ambos os grupos responderam a um questionário aplicado pela pesquisadora, após aceitação do TCLE e entrega do termo de confidencialidade. A pesquisa foi avaliada e aprovada sob o protocolo 176/2014 do Comitê de Ética da UNESC e pelo Comitê de Ética e pesquisa do Hospital São José.

Para a pesquisa, a amostra por conveniência foi composta por 35 pacientes, caracterizando-se como grupo-amostra. 0 grupo-controle foi composto por 35 pacientes. A abordagem total foi de 70 pacientes. Para este estudo, incluiu-se, no grupo-amostra, pacientes em tratamento oncológico com neoplasias de cabeça e pescoço, acima de 18 anos, que aceitaram e assinaram o TCLE. Já no grupo-controle, foram incluídos pacientes sem neoplasias de cabeça e pescoço, e pacientes acima de 18 anos com o TCLE assinado. Os pacientes abaixo de 18 anos, ou que não aceitaram assinar o TCLE, foram excluídos da pesquisa.

As variáveis abordadas neste trabalho incluem os fatores relacionados ao aparecimento de neoplasias de cabeça e pescoço. Pela literatura, essas variáveis podem ter induzido o desenvolvimento de tumor: idade, gênero, exposição ao sol, utilização de tabaco e álcool, HPV e histórico familiar.

Após a coleta de dados, elaborou-se um banco de dados no software StatisticalPackage for the Social Sciences (SPSS) versão 22, também utilizado para a análise estatística. Verificou-se a normalidade das variáveis numéricas pelo teste de Shapiro Wilk, que revelou distribuição normal para todas as variáveis numéricas, sendo utilizada a média e o desvio-padrão. Também estimouse a frequência absoluta (n) e relativa (\%) para as variáveis qualitativas.

Utilizou-se o teste T de Student (precedido do teste de Levene) para avaliar a média da idade em relação ao sexo e grupos dos pacientes do Hospital São José e UNESC; para todos os testes considerou-se um nível de significância a=0,05 e intervalo de confiança de $95 \%$. 


\section{RESULTADOS}

Analisou-se 70 pacientes, dos quais 35 tinham o diagnóstico de tumor de cabeça e pescoço, e o restante eram pacientes sem diagnóstico de tumor. Os questionários foram aplicados no período de março a maio de 2017.

Como pode ser visto na Tabela 1, no grupo-amostra 71,4\% eram homens e $28,6 \%$ eram mulheres, havendo uma prevalência do sexo masculino. Já o grupo-controle, compôs-se por $45,7 \%$ de homens e $54,3 \%$ de mulheres, havendo uma leve predileção pelo sexo feminino.

Tabela 1 - Perfil dos pacientes

\begin{tabular}{|c|c|c|}
\hline Variável & Hospital & UNESC \\
\hline \multicolumn{3}{|l|}{ Sexo } \\
\hline Feminino & $28,6 \%$ & $54,3 \%$ \\
\hline Masculino & $71,4 \%$ & 45,7 \\
\hline Idade Média & 62,49 anos & 51,60 anos \\
\hline \multicolumn{3}{|l|}{ Raça } \\
\hline Caucasiano & $94,3 \%$ & $82,9 \%$ \\
\hline Negro & $2,9 \%$ & $5,7 \%$ \\
\hline Pardo & $2,9 \%$ & $11,4 \%$ \\
\hline
\end{tabular}

Fonte: Dados da pesquisa

A idade dos pacientes variou de 30 a 88 anos no grupo-amostra, sendo que a idade média observada foi de 62,49 anos. Já no grupo-controle, a idade dos pacientes variou entre 30 e 67 anos, com uma média de 51,60 anos. (Tabela 1)

Houve uma predominância de pacientes caucasianos, sendo 94,3\% do grupo-amostra e $82,9 \%$ do grupo-controle. (Tabela 1)

Dos pacientes entrevistados, no grupo-amostra 76,4\% já foram fumantes em alguma época de sua vida, e no grupo-controle 56,3\% afirmam já ter feito uso contínuo de tabaco em alguma época. Em relação ao tempo de uso do cigarro, no grupo-amostra 46,2\% afirmaram fazer uso do tabaco de 30-40 anos e 15,4\% há 50 anos ou mais. Já no grupo-controle $22,2 \%$ de $30-40$ anos e $0,0 \%$ há 50 anos ou mais. (Tabela 2)

Tabela 2 - Fumantes

\begin{tabular}{|c|c|c|}
\hline Variável & Hospital & UNESC \\
\hline Já foi fumante & $76,4 \%$ & $59,3 \%$ \\
\hline \multicolumn{3}{|l|}{ Quanto tempo } \\
\hline 0-10 anos & $15,4 \%$ & $22,2 \%$ \\
\hline 10-20 anos & $7,7 \%$ & $22,2 \%$ \\
\hline 20-30 anos & $15,4 \%$ & $33,3 \%$ \\
\hline 30-40 anos & $46,2 \%$ & $22,2 \%$ \\
\hline 50 anos ou mais & $15,4 \%$ & $0,0 \%$ \\
\hline
\end{tabular}


ISSN 1983-5183

Tabela 3 - Etilistas

\begin{tabular}{lll}
\hline \hline Variável & Hospital & UNESC \\
\hline Já foi etilista & $37,1 \%$ & $8,6 \%$ \\
Quanto tempo & & \\
$0-5$ anos & $7,7 \%$ & $0,0 \%$ \\
$10-20$ anos & $46,2 \%$ & $66,7 \%$ \\
$20-30$ anos & $30,8 \%$ & $0,0 \%$ \\
30 anos ou mais & $15,4 \%$ & $33,3 \%$ \\
\hline \hline
\end{tabular}

Fonte: Dados da pesquisa

Em relação ao álcool, pode-se ver na Tabela 3 que 37,1\% dos pacientes do grupo-amostra relataram já ter sido etilista em alguma época da sua vida. Já no grupo-controle, este número se mostrou diminuído, sendo 8,6\%. Em relação à duração de tempo usando tal substância, no grupo-amostra 46,2\% tiveram tal hábito de 10-20 anos e 15,4\% por 30 anos ou mais. Já no grupo-controle, $66,7 \%$ de $10-20$ anos e 33,3\% de 30 anos ou mais.

Com relação à exposição em excesso ao sol, no grupo-amostra $86,7 \%$ dos pacientes afirmaram tal hábito. Já no grupo-controle, apenas $44,1 \%$ relataram a presença de tal fator pré-oncogênico. No grupoamostra, $14,3 \%$ dos pacientes foram expostos ao sol por 20-30 anos, 50,0\% de 30 anos ou mais. Já no grupocontrole, $6,7 \%$ de $20-30$ anos e $13,3 \%$ de 30 anos ou mais. (Tabela 4)

Tabela 4 - Exposição ao sol

\begin{tabular}{|c|c|c|}
\hline Variável & Hospital & UNESC \\
\hline Exposição em excesso ao sol & $86,7 \%$ & $44,1 \%$ \\
\hline \multicolumn{3}{|l|}{ Quanto tempo } \\
\hline $0-5$ anos & $3,6 \%$ & $26,7 \%$ \\
\hline 5-10 anos & $3,6 \%$ & $13,3 \%$ \\
\hline 10-20 anos & $28,6 \%$ & $40,0 \%$ \\
\hline 20-30 anos & $14,3 \%$ & $6,7 \%$ \\
\hline 30 anos ou mais & $50,0 \%$ & $13,3 \%$ \\
\hline
\end{tabular}

Fonte: Dados da pesquisa

Tabela 5 - Hereditariedade e Histórico de HPV

\begin{tabular}{lll}
\hline \hline Variável & Hospital & UNESC \\
\hline Histórico de câncer na família & $77,1 \%$ & $65,7 \%$ \\
Histórico de HPV & $0,0 \%$ & $2,9 \%$ \\
\hline \hline
\end{tabular}

Fonte: Dados da pesquisa

Foi visto que $77,1 \%$ dos pacientes do grupo-amostra relataram histórico de câncer na família, já no grupo-controle 65,7\% relataram a mesma situação. (Tabela 5)

Em relação ao HPV, nenhum paciente do grupo-amostra relatou ter essa doença, e apenas 2,9\% do grupo-controle relataram tal achado. (Tabela 5) 
ISSN 1983-5183

\section{DISCUSSÃO}

Os tumores, de modo geral, estão cada vez mais presentes e cada vez mais a população adquire hábitos que influenciam no aparecimento de tal patologia. O Instituto Nacional de Câncer (INCA) estima que em 2016 tenham sido registrados 596 mil novos casos de câncer no Brasil.

O câncer de cabeça e pescoço tem sido associado na literatura com alguns fatores, principalmente o uso de tabaco, álcool, histórico de HPV, hereditariedade, idade avançada e a exposição ao sol'12, 13, 14, 15, 16 .

A amostra composta por pacientes com neoplasias de cabeça e pescoço, obtida no Hospital São José, mostrou maior prevalência de pessoas do gênero masculino, representando $71,4 \%$, o que corrobora um estudo que fala que, dos 177 pacientes com tumor de cabeça e pescoço, $88,14 \%$ eram do sexo masculino ${ }^{17}$. O grupo-controle, formado por pacientes em atendimento nas clínicas integradas da UNESC, que não apresentaram neoplasias de cabeça e pescoço, apontou que $45,7 \%$ eram do gênero masculino e $54,3 \%$ eram do gênero feminino, indicando haver uma discreta predileção pelo gênero feminino no grupo-controle. A maior prevalência da doença em homens justifica-se pelas questões sociais, sendo que os homens se encontram mais relacionados com fatores carcinogênicos, como o álcool e o tabaco, do que as mulheres ${ }^{18}$.

Llewellyn et al. ${ }^{19}$ (2004) demonstraram que, dos pacientes acometidos pelo câncer oral, foi visto que $47 \%$ das mulheres e $48 \%$ dos homens faziam uso de tabaco. De mesma forma, outro estudo relatou que $66 \%$ das mulheres e $83 \%$ dos homens eram tabagistas ${ }^{16}$.

A faixa etária dos indivíduos entrevistados variou entre 30 a 88 anos, havendo uma média de 62,49 anos no grupo-amostra, e variou entre 30 e 67 anos, tendo no grupo-controle uma média de 51,60.

Em um estudo com 427 pacientes com tumor de cabeça e pescoço, em um hospital universitário do Noroeste do Estado de São Paulo, a idade média foi de 61 anos20.

O maior índice de aparecimento de câncer dá-se acima dos 65 anos de idade, com uma estabilização entre 85 e 90 anos. Porém, o índice de mortalidade por câncer diminui logo após os 90 anos. Por isso, entende-se que há ação de uma seletividade de pessoas que detêm mecanismos de imunidade antitumoral menos influenciados pela faixa etária ${ }^{14}$.

Houve um predomínio de $94,3 \%$ de pacientes brancos (caucasianos) portadores de câncer de cabeça e pescoço, o que é compatível com a literatura, que exibe uma prevalência mais alta de pacientes caucasianos acometidos por essa doença ${ }^{21,22,23}$.

No grupo-amostra, $77,1 \%$ dos pacientes afirmaram ter histórico de câncer na família; já no grupocontrole, o histórico foi relatado por $65,7 \%$ dos entrevistados. Há evidências de que a herança familiar desempenhe um papel decisivo no seu aparecimento ${ }^{13}$. Entretanto, no câncer de boca, o risco relativo para pacientes que relatem casos na família é menor que nos cânceres de faringe ou laringe. 0 maior problema na interpretação desses dados é distinguir se a doença ocorre em função da hereditariendade ou do estilo de vida semelhante entre os familiares. Outra dificuldade em relacionar a hereditariedade com o aparecimento das neoplasias de cabeça e pescoço são os métodos utilizados na coleta de dados, onde as pesquisas utilizam os prontuários médicos, que nem sempre trazem todas as informações a respeito da hereditariedade dos pacientes. 
ISSN 1983-5183

Dos pacientes entrevistados, com diagnóstico de neoplasia de cabeça e pescoço, 2,9\% relataram ser fumantes atualmente, sendo que 46,2\% dos entrevistados já fizeram uso do tabaco por 30 a 40 anos. Além disso, 73,5\% foram fumantes em alguma determinada fase de sua vida. No grupo-controle, 80,0\% dos pacientes negaram o uso do tabaco, sendo que 20,0\% afirmaram usar tabaco atualmente. Com o diagnóstico de neoplasia a tendência é que os pacientes cessem o hábito de fumar, e isso pode explicar o porquê do número de pacientes que fumam atualmente ser maior no grupo-controle.

Na literatura, o hábito de fumar está associado ao aparecimento de neoplasias de cabeça e pescoço. Em uma pesquisa realizada com 162 pacientes com câncer no trato aerodigestivo superior, 88,2\% eram fumantes ${ }^{24}$.

O tabaco apresenta-se como um fator pré-oncogênico agressivo, pois possui mais de 60 substâncias carcinogênicas que o formam, sendo isso associado à elevada temperatura do cigarro 5 .

O Instituto Nacional do Câncer (INCA) cita o hábito de fumar como o principal responsável de óbitos por neoplasias no Brasil. Sendo que, em fumantes, o risco de morte por câncer de boca é trinta vezes mais elevado do que em pessoas que não fazem uso do tabaco.

É visto na literatura que, ao parar o hábito de fumar, diminui-se o risco, mas não se descarta a possibilidade do aparecimento de alguma neoplasia ${ }^{25}$.

Nesta pesquisa obteve-se um dado relativo que mostrou que houve uma menor prevalência de pacientes com neoplasias, de cabeça e pescoço, que fumaram mais de 40 anos, quando comparados a pacientes que fumaram por 30 a 40 anos. Esse fato pode estar relacionado a uma predisposição genética desses pacientes, ou os pacientes que fumaram por mais tempo podem ter desenvolvido uma neoplasia em outro lugar que não seja cabeça e pescoço, não entrando nesta amostra. Além disso, é importante salientar que se a idade média dos indivíduos do grupo-amostra foi de 62,49 anos, é pouco provável que tenham fumado por mais de 50 anos.

Dos múltiplos fatores que podem induzir o aparecimento de tumores de cabeça e pescoço, o tabaco e o álcool são considerados os hábitos de maior potencial carcinogênico. O etilismo é visto também como um dos principais fatores que podem fazer com que surja um tumor, vindo apenas depois do tabaco, e este se mostra intimamente ligado com o aparecimento de câncer de língua e assoalho de boca ${ }^{26}$.

Verificou-se que houve uma prevalência de 37,1\% de pacientes que eram etilistas no grupoamostra, sendo que 46,2\% fizeram uso contínuo de álcool de 20 a 30 anos. Já no grupo-controle houve um baixo índice de etilistas, representando 8,6\% de pacientes, em alguma época da vida.

Nos dias atuais encontram-se evidências suficientes em relação ao etilismo e a carcinogênese, mesmo que ainda não sejam claros os mecanismos do álcool para causar o tumor ${ }^{27}$.

Atribui-se muito a capacidade do álcool de causar neoplasias por um dos seus metabólitos, o acetaldeído, pois este pode causar mutações no DNA celular. Porém, há características específicas de cada indivíduo que definem uma alta velocidade de transformação do etanol em acetaldeído, e isso pode esclarecer por que algumas dessas pessoas têm câncer e outras não27. 
ISSN 1983-5183

Dos pacientes com neoplasias entrevistados no grupo-amostra, 86,7\% afirmaram que em alguma época da vida tiveram um alto índice de exposição à radiação solar, com mais de 5 horas de exposição por dia. Já no grupo-controle, apenas 44,1\% relataram exposição solar superior a 5 horas por dia.

Silva et al. ${ }^{11}$ (2006) descrevem que, em um estudo feito com 111 pescadores, de 8 comunidades pesqueiras de Florianópolis, que tinham exposição diária ao sol, foram relatados 48 casos de queilite actínica e 3 casos de leucoplasia; 4 pescadores tiveram suspeita de carcinoma epidermoide.

A radiação ultravioleta está intimamente ligada ao aparecimento do câncer bucal, especialmente de lábio inferior ${ }^{15}$.

A radiação solar, depois de um longo período, é capaz de fazer com que apareçam lesões de importância biológica. Pode-se tomar como exemplo a exposição solar frequente e superior a 15-30 anos, que faz com que haja modificações celulares, podendo evoluir para o tumor $^{10}$.

Dos pacientes entrevistados do grupo-amostra, nenhum relatou ter HPV; já no grupo-controle, apenas um paciente relatou ter tal patologia. Tal relato corrobora a literatura, que diz que muitos que possuem o vírus não sabem que o têm, pois este pode instalar-se no organismo e manter-se latente sem nenhuma manifestação durante longos períodos. Alguns dos tipos de HPV são capazes de persistir num espaço de tempo mais prolongado, permitindo assim que aconteçam alterações celulares, que acarretam o surgimento de verrugas genitais, papilomatose respiratória recorrente (PRR) e alguns tipos de neoplasias como câncer de colo de útero e orofaringe ${ }^{12}$.

Com o aumento da expectativa de vida e da alta tendência de mortalidade causada pelo câncer, observa-se a importância de reavaliar as formas de promoção e prevenção realizadas, de modo que a população e os profissionais sejam conscientizados sobre a importância do autoexame e a redução de hábitos como o tabagismo e o alcoolismo, por meio de programas educacionais que podem ser fundamentais para diminuir o número de novos casos de câncer, bem como diminuir o número de óbitos pelo mesmo.

\section{CONCLUSÃO}

Os dados obtidos com esta pesquisa foram compatíveis com a literatura atual, verificouse a presença de fatores pré-oncogênicos acentuados nos pacientes com neoplasia de cabeça e pescoço; já nos pacientes sem o diagnóstico de tal patologia, houve um baixo índice desses mesmos fatores.

Das variáveis obtidas neste estudo, em relação aos pacientes com tumor, as que se mostraram mais relevantes foram: o uso em demasia de tabaco e álcool, anos de exposição ao sol, histórico de câncer na família, a idade elevada e a cor.

Tendo em vista as proporções desse problema de saúde pública e o sombrio prognóstico dessa doença, fazem-se necessárias campanhas de prevenção para que diminuam os fatores de risco e que haja medidas para um diagnóstico precoce de tal patologia. 
ISSN 1983-5183

\section{REFERÊNCIAS}

1. GALBIATTI AL, Padovani-Junior JA, Maniglia JV, Rodrigues CD, Pavarino EC, GoloniBertollo EM. Head and neck cancer: causes, prevention and treatment. Brazilian journal of otorhinolaryngology 2013 Mar-Apr;79(2):239-47.

2. JEMAL A, Bray F, Center MM, Ferlay J, Ward E, Forman D. Global cancer statistics. CA: $a$ cancer journal for clinicians 2011 Mar-Apr;61(2):69-90.

3. LOTHAIRE P, De Azambuja E, Dequanter D, Lalami Y, Sotiriou C, Andry G, et al. Molecular markers of head and neck squamous cell carcinoma: promising signs in need of prospective evaluation. Head \& neck 2006 Mar;28(3):256-69.

4. PITOT HC, Dragan YP. Facts and theories concerning the mechanisms of carcinogenesis. FASEB journal : official publication of the Federation of American Societies for Experimental Biology 1991 Jun;5(9):2280-6.

5. OGDEN GR. Alcohol and oral cancer. Alcohol (Fayetteville, NY) 2005 Apr;35(3):169-73.

6. LIANG C, Marsit CJ, Houseman EA, Butler R, Nelson HH, Mcclean MD, et al. Gene-environment interactions of novel variants associated with head and neck cancer. Head \& neck 2012 Aug;34(8):1111-8.

7. DIX D, Cohen P, Flannery J. On the role of aging in cancer incidence. Journal of theoretical biology 1980 Mar 7;83(1):163-73.

8. GILLISON ML, Shah KV. Chapter 9: Role of mucosal human papillomavirus in nongenital cancers. Journal of the National Cancer Institute Monographs 2003 31):57-65.

9. KOJIMA A, Maeda H, Sugita Y, Tanaka S, Kameyama Y. Human papillomavirus type 38 infection in oral squamous cell carcinomas. Oral oncology 2002 Sep;38(6):591-6.

10. SPITZ MR. Epidemiology and risk factors for head and neck cancer. Seminars in oncology 1994 Jun;21(3):281-8.

11. SILVA FD, Daniel FI, Grando LJ, Calvo MC, Rath IBS, Fabro SML. Estudo da prevalência de alterações labiais em pescadores da ilha de Santa Catarina. Rev Odonto Ciência 2006 21(51):jan./mar. .

12. BRASIL. Guia prático sobre o HPV. Brasília: Ministerio da Saúde; 2014 [Acesso em: 29 abril. 2019.]; Disponível em: http://portalarquivos.saude.gov.br/campanhas/2014/hpv/ Guia_perguntas_e_repostas_MS_HPV_profissionais_de_saude.pdf.

13. HASSANEIN KA, Musgrove BT, Bradbury E. Psychological outcome of patients following treatment of oral cancer and its relation with functional status and coping mechanisms. Journal of cranio-maxillo-facial surgery : official publication of the European Association for Cranio-Maxillo-Facial Surgery 2005 Dec;33(6):404-9.

14. CARUSO C, Lio D, Cavallone L, Franceschi C. Aging, longevity, inflammation, and cancer. Annals of the New York Academy of Sciences 2004 Dec;1028(1-13.

15. WERNER JE, Fontanella V. Perfil epidemiológico dos pacientes portadores de câncer bucal atendidos no Hospital Santa Rita, Porto Alegre/RS. Stomatos (Brasil) Num28 Vol15 2009 jan./jun.;15(28):3-16. 
ISSN 1983-5183

16. SANTOS GL, Freitas VS, Andrade MC, Oliveira MC. Fumo e álcool como fatores de risco para o câncer bucal. Odontol Clín-Cient 2010 abr./jun.;9(2):131-3.

17. FIGUEIREDO AM. Prevalências e características clínico-epidemiológicas do câncer bucal e de cabeça e pescoço no Hospital Estadual de Bauru/SP [Dissertação]. Bauru: Universidade de São Paulo, Faculdade de Odontologia de Bauru; 2013.

18. OLIVEIRA DT, Oddel EW. Diagnóstico precoce e prevenção do câncer de boca. In: Buischi, YP. Promoção de saúde bucal na clínica odontológica. São Paulo: Artes Médicas; 2000.

19. LLEWELLYN CD, Johnson NW, Warnakulasuriya KA. Risk factors for oral cancer in newly diagnosed patients aged 45 years and younger: a case-control study in Southern England. Journal of oral pathology \& medicine : official publication of the International Association of Oral Pathologists and the American Academy of Oral Pathology 2004 Oct;33(9):525-32.

20. AlVARENGA LM, Ruiz MT, Pavarino-Bertelli EC, Ruback MJ, Maniglia JV, Goloni-Bertollo M. Epidemiologic evaluation of head and neck patients in a university hospital of Northwestern Sao Paulo State. Brazilian journal of otorhinolaryngology 2008 Jan-Feb;74(1):68-73.

21. WUNSCH-FILHO V. The epidemiology of oral and pharynx cancer in Brazil. Oral oncology 2002 Dec;38(8):737-46.

22. CHOI KK, Kim MJ, Yun PY, Lee JH, Moon HS, Lee TR, et al. Independent prognostic factors of 861 cases of oral squamous cell carcinoma in Korean adults. Oral oncology 2006 Feb;42(2):208-17.

23. BERTO JC, Rapoport A, Lehn CN, Cestari Filho GA, Javaroni AC. Relação entre o estadiamento, o tratamento e a sobrevida no câncer da faringe. Rev Col Bras Cir 2006 ago.;33(4):207-10.

24. SANTOS RA, Portugal FB, Felix JD, Santos PMO, Siqueira MM. Avaliação epidemiológica de pacientes com câncer no trato aerodigestivo superior: relevância dos fatores de risco álcool e tabaco. Rev bras cancerol 2012 jan.-mar. ;58(1):21-9.

25. KUMAR B, Cordell KG, Lee JS, Worden FP, Prince ME, Tran HH, et al. EGFR, p16, HPV Titer, $\mathrm{BCl}-\mathrm{xL}$ and $\mathrm{p53}$, sex, and smoking as indicators of response to therapy and survival in oropharyngeal cancer. Journal of clinical oncology: official journal of the American Society of Clinical Oncology 2008 Jul 1;26(19):3128-37.

26. REIS SRA, Lima CR, Marchionni AMT, Setubal MG. Fatores de risco do câncer da cavidade oral e da orofaringe. I. fumo, álcool e outros determinantes. RPG rev pos-grad 1997 abr.-jun. ;4(2):127-32.

27. INTERNATIONAL Agency for Research on Cancer. Monographs on the evaluation of the carcinogenic risck of chemicals to humans. Alcoholdrinking 1988 44(1):83-126.

RECEBIDO EM 01/03/2018

ACEITO EM 21/01/2019 